\title{
Computation of Neutral Gas Flow From a Hall Thruster Into a Vacuum Chamber
}

\author{
Iain D. Boyd, Chunpei Cai, Mitchell L. R. Walker and Alec D. Gallimore \\ Department of Aerospace Engineering, University of Michigan, Ann Arbor, MI 48109
}

\begin{abstract}
The finite pressure present in vacuum chamber testing of space propulsion systems such as Hall thrusters can have a number of undesirable effects. For example, the thrust generated by the thruster is higher and the plume divergence angle larger in ground tests in comparison to space operation. To try to quantify these effects, the direct simulation Monte Carlo method is applied to model a cold flow of xenon gas expanding from a Hall thruster into a vacuum chamber. The simulations are performed for the P5 Hall thruster operating in a large vacuum tank at the University of Michigan. Comparison of the simulation results is made with experimental measurements of pressure obtained with a series of ion gauges. The mass flow rate through the thruster and the total pumping speed of the vacuum chamber are varied. A key physical parameter in the simulations concerns the probability that a xenon atom incident on a cryogenic pumping panel actually sticks to the panel. For a reasonable range of values for the sticking coefficient, excellent agreement between simulation and experiment is obtained for several different conditions.
\end{abstract}

\section{INTRODUCTION}

It is technically challenging and expensive to create the on-orbit environment in a ground-based laboratory facility. All ground-based vacuum facilities possess a low-density background neutral gas due to physical pumping limitations and the leak rate of the facility. As gas is introduced into the vacuum chamber in the form of propellant, the background pressure rises until the pumping speed, facility leak rate, and propellant flowrate equilibrate for the operating condition. The facility background gas present in the vacuum chamber can distort the exhaust plume of the thruster [1]. High-energy exhaust particles interact with the neutral background particles through charge exchange collisions (CEX) [2]. In the plume, the effects of CEX products are most evident in the perimeter, where they lead to an increase in the measured current density. Thruster operation and performance are dependent on the background pressure of the facility [3]. At elevated background pressures, residual gas particles can be entrained into the thruster discharge region artificially increasing engine thrust. Elevated facility pressure has also been found to increase the width of the ion energy distribution function through elastic collisions between beam ions and neutral background particles [4]. Due to these effects, the validity of comparisons made between data taken in facilities with different background pressures, especially at $10^{-4}$ Torr and higher, is questionable. However, comparisons have been made between data taken in Russia and NASA [5]. In order to correlate data taken in ground based facilities to in-space thruster performance, the effect of facility background pressure on thruster operation must be fully characterized and taken into account when analyzing test data. Because of the drastic implications of facility effects, electric propulsion technology has reached the point where standard guidelines must be developed for test facilities to ensure reliable engine development and testing. This need has become even more pressing now that very high power Hall thrusters are being developed [6].

Several investigations are underway to numerically model thruster performance and the interactions between Hall thrusters and spacecraft [7,8]. The results of these models are highly dependent on experimentally measured boundary conditions. One of the most important auxiliary inputs required by these codes is background pressure of a laboratory vacuum chamber [7].

The University of Michigan's Plasmadynamics and Electric Propulsion Laboratory (PEPL) has launched an investigation of facility effects introduced by elevated backpressures. This investigation has thus far included 
measuring the performance of the P5 Hall thruster at different pumping speeds [9], an evaluation of a collimated Faraday probe's ability to filter out CEX ions while measuring the ion current density at elevated back pressures $[2,10]$, and a comparison of NASA Glenn Research Center's (GRC) and Jet Propulsion Laboratory's (JPL) nude Faraday probes [11].

The objective of a recent P5 experiment presented in Ref. 12 was to create a pressure map inside a large vacuum chamber at a series of cold anode flow rates corresponding to typical P5 Hall thruster operating conditions at different facility pumping speeds. This is the first step in creating a technique for making neutral density pressure maps with hot flow in a Hall thruster facility. In the current investigation, the results of the cold flow pressure map are compared to a numerical simulation of the chamber in order to develop the tools that will be necessary to correct for facility effects.

In the following, the vacuum chamber, Hall thruster, and measurement technique are first described. Then, a brief description of the numerical model is presented. Direct comparison is then made between simulation and measurement for the pressure map in the vacuum chamber for a number of different cold flow configurations. Numerical results for a single hot-flow operating point are also provided. Finally, some conclusions and directions for future work are offered.

\section{EXPERIMENTAL STUDY}

\section{Vacuum Facility}

All experiments were conducted in the University of Michigan's Large Vacuum Test Facility (LVTF), shown schematically in Fig. 1. The thruster is mounted at thruster station 1. The LVTF is a stainless steel clad vacuum chamber that has a diameter of $6 \mathrm{~m}$ and a length of $9 \mathrm{~m}$. Two 2,000 CFM blowers and four 400 CFM mechanical pumps evacuate the LVTF to moderate vacuum (30 - 100 mTorr). To reach high vacuum the LVTF is equipped with seven CVI TM-1200 re-entrant cryopumps, each of which is surrounded by a $\mathrm{LN}_{2}$ baffle. The combined pumping speed of the facility is $500,000 \mathrm{l} / \mathrm{s}$ on air, and $240,000 \mathrm{l} / \mathrm{s}$ on xenon with a base pressure of $2.5 \times 10^{-7} \mathrm{Torr}$. The cryopump system can be operated with any number of pumps in use. For the experiments reported here, the LVTF was operated with seven cryopumps. At the average anode flow rates investigated-5.25, 10.46, and 14.09 $\mathrm{mg} / \mathrm{s}$, all with a $0.60 \mathrm{mg} / \mathrm{s}$ cathode flow-and at a nominal xenon pumping speed of 240,000 1/s, the operating pressures of the LVTF were approximately $1.7 \times 10^{-6}, 7.7 \times 10^{-6}$, and $1.0 \times 10^{-5}$ Torr on xenon.

Chamber pressure was monitored by two hot-cathode ionization gauges, as indicated in Fig. 1. The first gauge was a Varian model Bayard-Alpert (BA) gauge with a HPS model 919 Hot Cathode Controller. The BA model 571 ionization gauge is connected to the chamber via a $25-\mathrm{cm}-\mathrm{long}$, by 3.48 -cm-inner-diameter, tube. The second is a Varian model UHV-24 nude gauge with a Varian UHV senTorr Vacuum Gauge Controller. This unit was calibrated on air by the manufacturer. Pressure measurements from both gauges were corrected for xenon using the known base pressure on air and a correction factor of 2.87 for xenon according to the following equation [13]:

$$
P_{c}=\frac{P_{i}-P_{b}}{2.87}+P_{b}
$$

where $\mathrm{P}_{c}$ is the corrected pressure on xenon, $\mathrm{P}_{\mathrm{b}}$ is the base pressure, and $\mathrm{P}_{\mathrm{i}}$ is the indicated pressure when xenon is flowing into the vacuum chamber. The corrected average pressure of the two gauges is normally reported as the chamber background pressure. All pumping speeds and pressures reported in the following are corrected for xenon. 


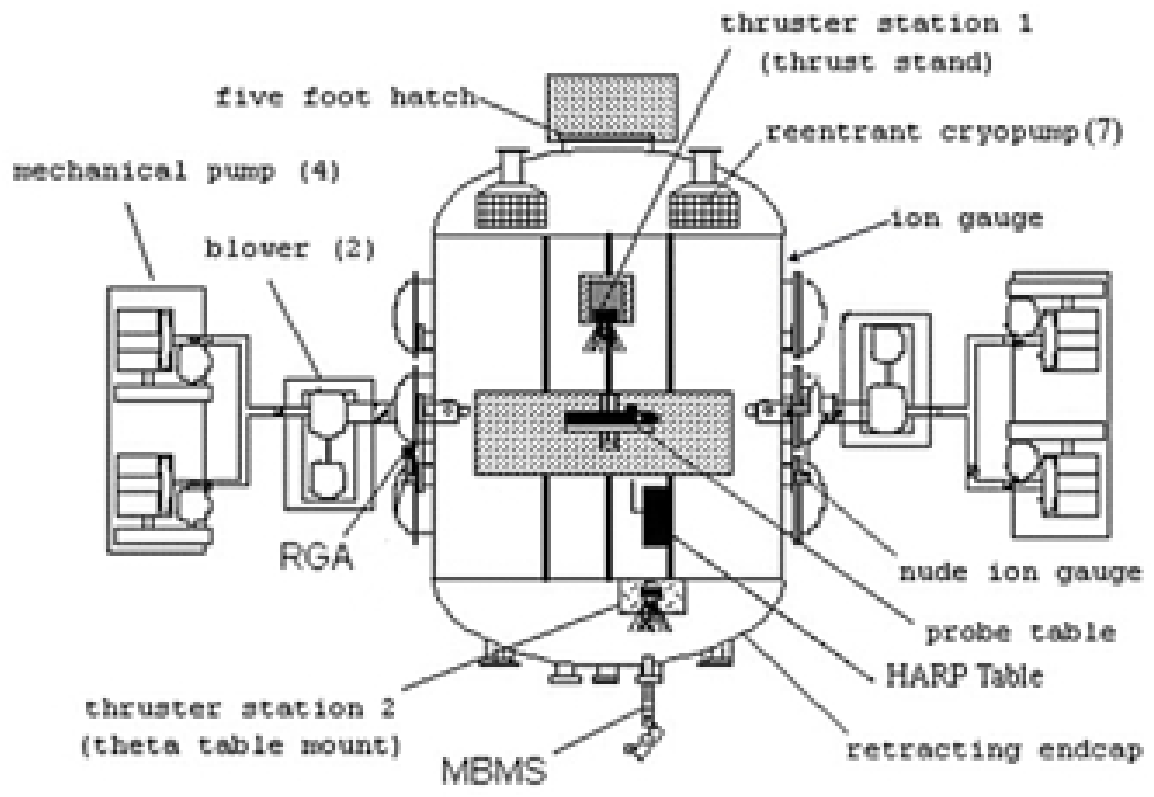

FIGURE 1. Schematic diagram of the LVTF.

\section{Hall Thruster}

All experiments are performed on the P5-2 (also known as the NASA-173M) $5 \mathrm{~kW}$ laboratory-model Hall thruster. A more detailed discussion of the thruster can be found in Ref. 9. This thruster is designed to operate in both singleand two-stage modes. For these experiments, the electrode used for two-stage operation was replaced with the same ceramic used in the chamber walls, enabling the engine to be operated as a single-stage device. The NASA-173M has a mean diameter of $148 \mathrm{~mm}$, a channel width of $25 \mathrm{~mm}$, and has a nominal power rating of $5 \mathrm{~kW}$ [9]. For these experiments, xenon was flowed through the thruster anode and no plasma discharge was present.

A NASA GRC laboratory-model hollow cathode was located at the 12 o'clock position on the thruster. The cathode orifice was located approximately $25 \mathrm{~mm}$ downstream and $25 \mathrm{~mm}$ radially away from the outer front pole piece at an inclination of $30^{\circ}$ from the thruster centerline. Xenon flow rates corresponding to the necessary flow rates of the operating NASA-173M Hall thruster were set on the cathode. This will yield comparable backpressures for future pressure maps with the NASA-173M Hall thruster operating in hot-flow mode.

High-purity (99.999\% pure) xenon propellant was supplied to the Hall thruster from compressed gas bottles through stainless-steel feed lines. Anode and cathode propellant flows were controlled and monitored with MKS 1179A mass flow controllers. The flow controllers were calibrated with a custom apparatus that measures gas pressure and temperature as a function of time in an evacuated chamber of known volume. The mass flow controllers have an accuracy of $\pm 1 \%$ full scale.

\section{Ionization Gauges}

The BA hot-cathode ionization gauge accurately measures pressure over the range of $10^{-4}$ to $10^{-12}$ Torr [10]. Estimates of the pressure for the experiment were $10^{-5}$ to $10^{-8}$ Torr, based on previous experimental data. Because of its accuracy over the anticipated range of pressures, the BA gauge was selected to measure the chamber pressure field.

Five Varian 571 BA type standard range ionization gauge tubes were used to measure the chamber pressure field because of their rugged construction, low cost, and long life. Future work at PEPL will include a hot flow pressure map of the LVTF. Thus, the BA gauges need a neutralizer to ensure that the plasma does not affect pressure 
measurements, per Varian's recommendation. To make the hot and cold flow experiments identical in setup, the neutralizers were present during the cold flow experiment. The neutralizer design prevents plume ions from having a direct line of sight to the ionization gauge filament. The neutralizer contains two 72 mesh screens $(0.5$ by $0.5 \mathrm{~mm}$ and 1.0-mm-thick) to ensure neutralization of any ions that travel inside the orifice and that are not neutralized by wall collisions.

Calibration of the five ionization gauge systems was performed by the NASA GRC Calibration Laboratory. Each system, comprising of a BA gauge, the actual internal and external cables used in the LVTF mapping, a Varian 10wire vacuum chamber instrumentation feedthrough, and a Varian BA circuit board mounted in either the senTorr or Multi-Gauge controller, was calibrated with nitrogen as a one-piece unit using a National Institute of Standards and Technology traceable spinning rotor gauge.

To generate the two-dimensional mapping inside the vacuum chamber, we mounted the ionization gauges to a custom built, two-axis positioning stage developed by New England Affiliated Technologies (NEAT). The positioning system is composed of a 1.8 -m-long linear stage in the radial direction that is mounted on a 0.9 -m-long axial stage with an absolute linear position accuracy of $0.15 \mathrm{~mm}$. A LabView VI controlled the motion of the two linear position tables, which in turn moved the Ionization Gauge Positioning System (IGPS) that carried the five BA gauges used to survey the chamber. The IGPS allowed the pressure measurements to be taken throughout the majority of the chamber with a single evacuation cycle of the LVTF. The area mapped by the IGPS encompasses an area with a minimum distance from the thruster of $0.5 \mathrm{~m}$, encompassing the typical $1 \mathrm{~m}$ distance plume properties are measured.

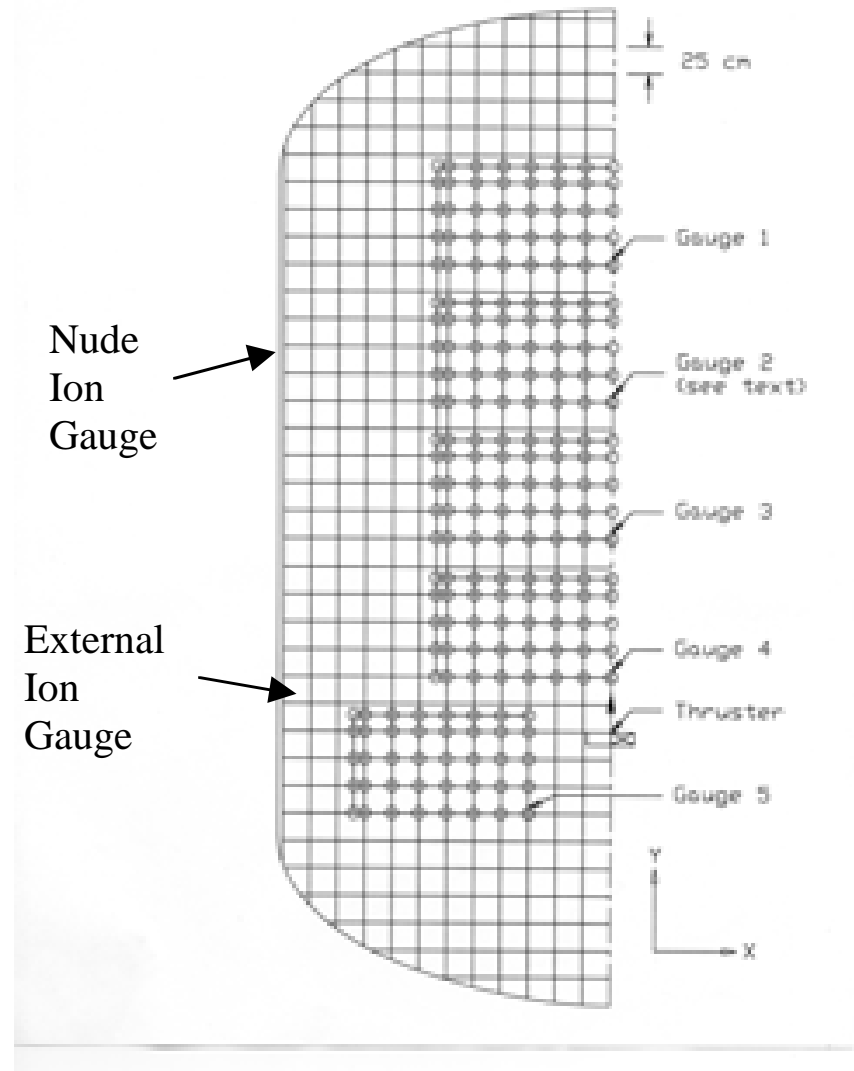

FIGURE 2. LVTF half-plane (looking down) with the IGPS and a $25 \mathrm{~cm}$ X $25 \mathrm{~cm}$ grid. Each open circle denotes the location of a data point. 
Figure 2 displays the LVTF and the $25 \mathrm{~cm} \mathrm{X} 25 \mathrm{~cm}$ grid on which data points were taken. The solid circles indicate the position of each of the five probes when the IGPS is in the initial position. The note in Fig. 2 denotes that gauge 2 is positioned on the opposite side of the chamber centerline to avoid being immersed in any wake effects created by gauge 3 . Figure 2 also shows the coordinate system used for this experiment and the numerical simulation. The coordinate system origin is located at the discharge chamber exit plane on the thruster centerline, where negative $\mathrm{X}$ is to the left and positive $\mathrm{Y}$ is up.

\section{NUMERICAL STUDY}

Hall thrusters primarily use xenon as propellant. The xenon plasma plume from a Hall thruster is composed of beam ions with velocities on the order of $16 \mathrm{~km} / \mathrm{s}$, low energy charge exchange ions, neutral atoms, and electrons. The total number density is of the order of $10^{18} \mathrm{~m}^{-3}$ that places the plasma in the rarefied flow regime. Computational analysis of Hall thruster plumes is regularly performed using a hybrid DSMC-PIC formulation, e.g. see Refs. 14, 15, 16. The direct simulation Monte Carlo (DSMC) method [17] models the collisions of the heavy particles (ions and atoms). The Particle In Cell (PIC) method [18] models the transport of the ions in electric fields. A hybrid approach is used in which the electrons are modeled using a fluid description.

In the present study, we apply an existing axially symmetric PIC-DSMC code developed specifically for Hall thruster plumes [16]. The flow conditions allow the assumption of charge neutrality. Therefore, in each computational cell, the number of particles representing ions (both $\mathrm{Xe}^{+}$and $\mathrm{Xe}^{2+}$ are included) specifies the electron density. The Boltzmann relation, a simplified form of the electron momentum equation (for the case of collisionless, un-magnetized, isothermal, currentless electrons), is then used to calculate the plasma potential for the cell. Spatial differentiation of the potential provides the electric field. The field is then applied to modify the velocity of the ion particles. There are two collision mechanisms that are important in Hall thruster plumes: (a) momentum exchange; and (2) charge exchange. Momentum exchange is calculated for atom-atom and atom-ion interactions. Atom-atom collisions employ the Variable Hard Sphere (VHS) model [17] and atom-ion collisions employ a model due to Dalgarno et al. [19]. In each case, isotropic scattering is assumed. In the charge exchange collisions, cross sections measured experimentally by Miller et al. [20] are employed and it is assumed that no scattering occurs.

The Large Vacuum Test Facility (LVTF) operated at the University of Michigan can be represented as a cylinder of length $9 \mathrm{~m}$ and diameter $6 \mathrm{~m}$. The DSMC-PIC computations employ a grid of 91 by 61 uniform, rectangular cells. At steady state, the computations typically employ 200,000 particles and the total run time for each case is on the order of four hours. In the cold-flow simulations, the PIC steps are of course not applied. The walls of the chamber are modeled assuming fully diffuse reflection at a temperature of $300 \mathrm{~K}$. The LVTF is equipped with seven cryogenic pumps that are grouped into two sets: (1) four side pumps with a total pumping area of about $4.15 \mathrm{~m}^{2}$; and (2) three top pumps with a total pumping area of $3.11 \mathrm{~m}^{2}$. In the simulations, the total area of each of these two sets is represented by a single pumping surface located in the vicinity of the actual pumps. The temperature of the pumping surfaces is assumed to be $15 \mathrm{~K}$. There are no data for the sticking coefficient of xenon on cryo-genic panels, however, data for other noble gases indicate a range of 0.6 to 0.8 [21]. Simulations are therefore performed with values of $0.6,0.8$, and 1.0 to study the sensitivity of the results to this unknown parameter.

\section{RESULTS}

In the following, results are presented for several different cold flow conditions of the Hall thruster in which the plasma is not ignited. These flows therefore simply consist of neutral xenon atoms. The simulation results are compared directly with experimental measurements of pressure for a number of conditions in which the mass flow rate and pumping speed are varied. A single hot-flow case is also presented, although there is not yet any experimental data for this case. 


\section{Cold Flow}

Simulation results are presented in Figs. $3 \mathrm{a}$ and $3 \mathrm{~b}$ for a mass flow rate of $10.46 \mathrm{mg} / \mathrm{s}$ and a total pumping speed of $240,000 \mathrm{l} / \mathrm{s}$ (all seven pumps operating). The experiments included flow of xenon through the cathode of $0.6 \mathrm{mg} / \mathrm{s}$ to mimic hot-flow operation and this flow rate was added in the simulation to the flow from the thruster. Figure $3 \mathrm{a}$ compares the simulation results obtained using a sticking coefficient of 0.6 for chamber pressure (upper half) with the measured data (lower half). Note that the ion gauges cannot be used within $50 \mathrm{~cm}$ of the thruster. The localized reductions in pressure in the simulations are at the locations of the side and top pumps. Clearly, very good agreement is obtained between the simulations and the measurements.

Figure $3 \mathrm{~b}$ shows the data comparison along the thruster centerline for the three different values of the sticking coefficient. The simulation results are sensitive to this parameter, although the profiles obtained with values of 0.6 or 0.8 show arguably more acceptable agreement with the measured data.
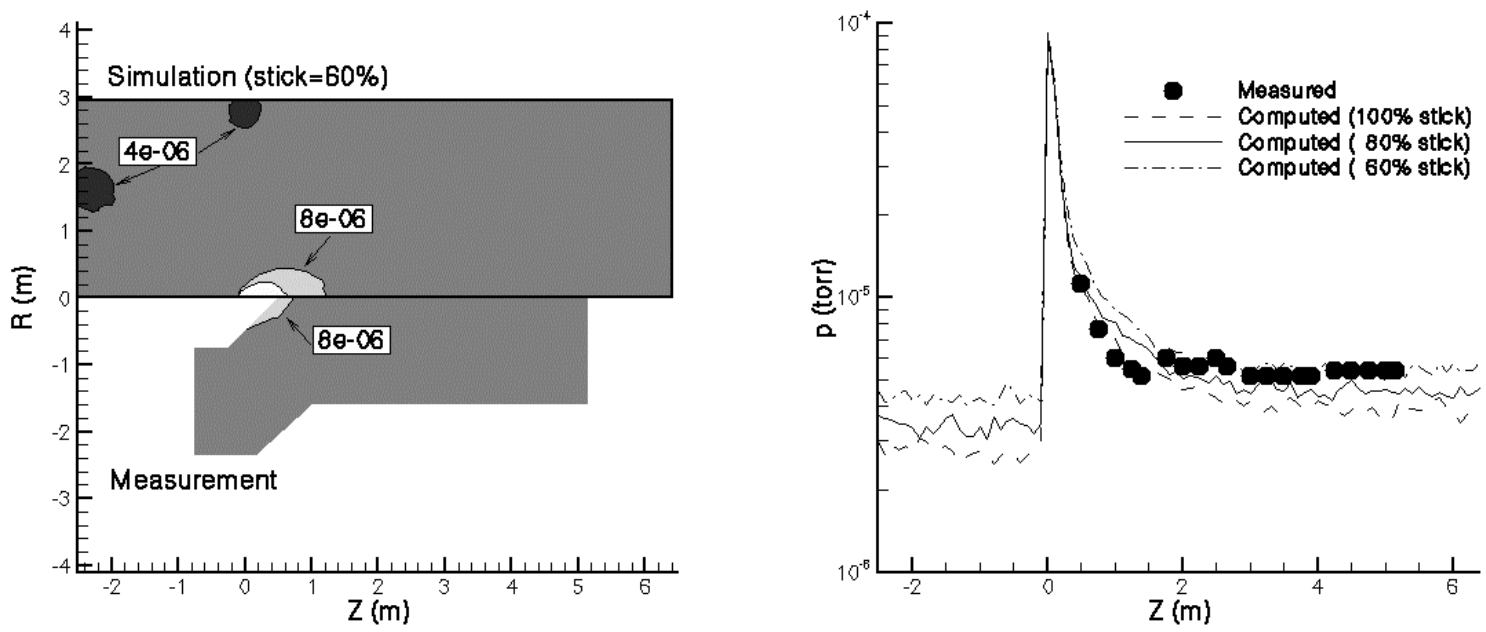

FIGURE 3. Comparisons of simulated and measured pressure distribution within the LVTF for cold flow operation of the P5 Hall thruster at $10.46 \mathrm{mg} / \mathrm{s}$ flow rate and 240,000 1/s pumping: (a) overall flow field; (b) along the thruster centerline.
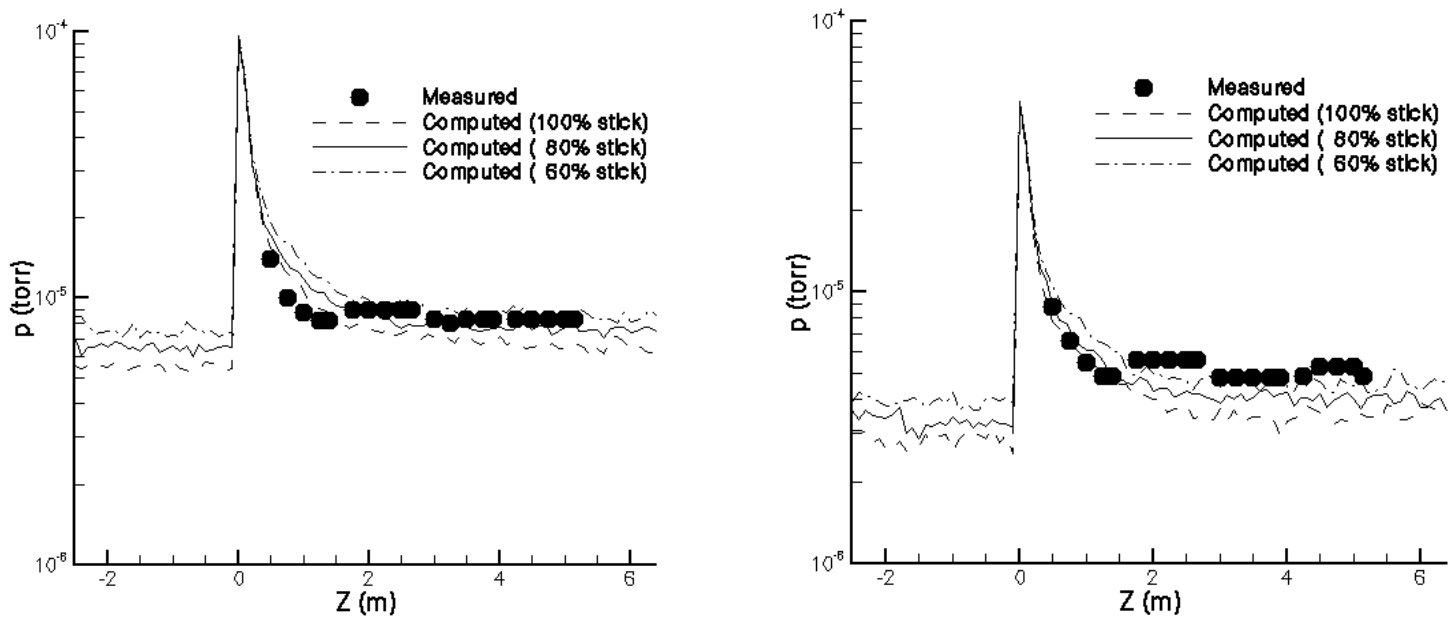

FIGURE 4. Comparisons of simulated and measured pressure distribution within the LVTF for cold flow operation of the P5 Hall thruster: (a) at $10.46 \mathrm{mg} / \mathrm{s}$ and 140,000 1/s; (b) at $5.25 \mathrm{mg} / \mathrm{s}$ flow rate and 140,000 1/s.

To investigate the generality of the performance of the simulations, two additional cases are simulated. The first retains the flow rate of $10.46 \mathrm{mg} / \mathrm{s}$ and considers the lower pumping rate of 140,000 1/s by turning off the three side 
pumps in the simulation. These results are shown in Fig. 4a. In Fig. 4b, the results are shown for a flow rate of 5.25 $\mathrm{mg} / \mathrm{s}$ (again with $0.6 \mathrm{mg} / \mathrm{s}$ flowing through the cathode) with a total pumping of 140,000 1/s. In both Figs. 4a and $4 \mathrm{~b}$, excellent agreement is obtained between simulation and experiment.

\section{Hot Flow}

The conditions at the exit plane of the P5 Hall thruster are estimated using existing measured data of thruster performance, ion current, fraction of multiple charges, and flow rate [22]. Once again, a key parameter in the simulation is the sticking coefficient of atoms and ions on the cryo-panels. In the region of the chamber where the pumps are located, only low energy charge exchange ions will appear. Therefore, it is assumed that the same value of sticking coefficient can be applied to both atoms and ions. In the absence of experimental data, a single simulation is performed using a sticking coefficient of 1.0 for a flow rate of $5.33 \mathrm{mg} / \mathrm{s}$ (with an additional $0.6 \mathrm{mg} / \mathrm{s}$ of neutral atom flow to mimic the cathode flow) and a pumping speed of 140,000 1/s (corresponding to the cold flow case shown in Fig. 4b). Under these conditions, at a voltage of $300 \mathrm{~V}$, the P5 produces a thrust of $86 \mathrm{mN}$, a discharge current of $4.86 \mathrm{~A}$, and a fraction of $\mathrm{Xe}^{2+}$ of 0.231 [22]. The ion temperature is assumed to be $4 \mathrm{eV}$ and the atom temperature is assumed to be $1000 \mathrm{~K}$. As the beam ions have a significant directed velocity (about $16 \mathrm{~km} / \mathrm{s}$ ) the simulation time-step is reduced by an order of magnitude in comparison to that employed in the cold flow simulations. This requires the simulation to be run for 2,000,000 iterations to ensure steady state. A total of about 300,000 particles are used and the simulation time is about 60 hours.

The overall pressure fields of the hot and cold flow simulations at $5.33 \mathrm{mg} / \mathrm{s}$ and 140,000 1/s are compared in Fig. 5a. The hot flow has the expected shape for a Hall thruster plume. Note that the pressures on axis are much higher in the hot flow in comparison to the cold flow. This behavior is mainly explained by very high ion temperatures computed in the hot flow case. The velocity distribution function of the ions in the plume is made up of two components: beam ions at a high directed velocity and an assumed temperature of about $4 \mathrm{eV}$, and charge exchange ions with a considerably smaller velocity and temperature. Under these nonequilibrium conditions, a temperature is ill-defined. From kinetic theory, the temperature is proportional to the width of the velocity distribution and this produces the very high values computed under these conditions. In all the DSMC-PIC results, pressure is calculated from temperature using the ideal gas law. Clearly, direct comparison of simulation results with experiments will require a much more careful treatment of these effects. In the absence of experimental data, this important issue is left to a later stage. In Fig. 5b, comparisons are made between hot flow simulation data and cold flow simulation and measured data along the thruster centerline. The total pressure in the hot-flow case (including both ions and atoms) is significantly higher than the cold flow data due to the elevated ion temperatures. The pressure computed only from the atoms in the hot flow case is also shown and is similar to the profile from the cold flow case.
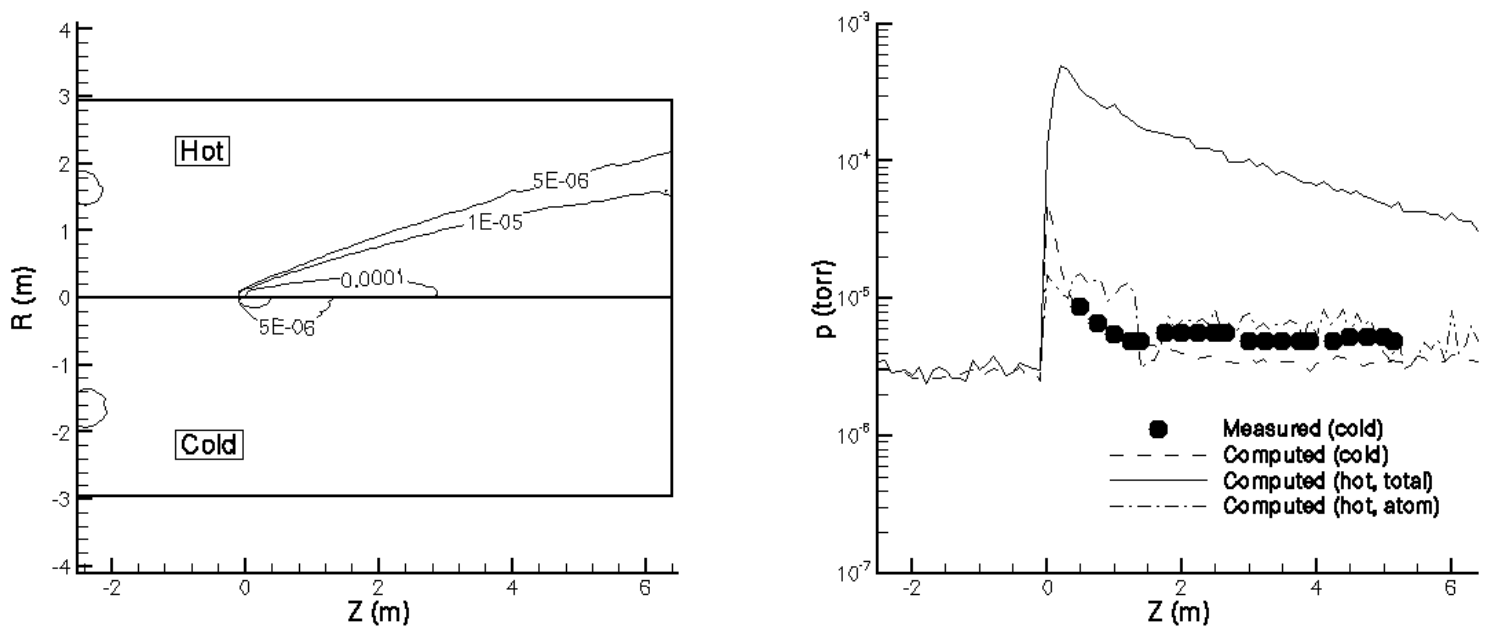

FIGURE 5. Comparisons of simulated pressure distributions within the LVTF for hot and cold flow operation of the P5 Hall thruster at $5.33 \mathrm{mg} / \mathrm{s}$ flow rate and 140,000 1/s pumping: (a) overall flow field; (b) along the thruster centerline. 


\section{CONCLUDING REMARKS}

Simulations were performed for three different cold flow cases of xenon exhausted by the P5 Hall thruster into the LVTF chamber at the University of Michigan. Thruster mass flow rate and facility total pumping speed were varied between the simulations. Direct comparisons for these cases were made with experimental data taken recently in the LVTF using ion gauges. While the simulation results were found to be sensitive to the sticking coefficient assumed for interaction between the xenon atoms and the cryogenic pumps, very good agreement was obtained between the simulation and the measured data. A single hot flow case was simulated at a mass flow rate and pumping speed that was also investigated in cold flow mode. The pressures simulated on axis for the hot flow case were considerably larger than those computed under cold flow conditions. This behavior is attributed to very high ion temperatures predicted by the simulation that result from the poor definition of temperature in a highly nonequilibrium environment. When hot flow data become available, care will have to be taken to perform a more accurate estimation of the pressure in this environment.

\section{ACKNOWLEDGMENTS}

Two of the authors (IDB and CC) gratefully acknowledge the financial support for this work from the Air Force Research Laboratory. The experimental research was supported by the Air Force Office of Scientific Research through grants F49620-00-1-0201 and F49620-01-1-0061.

\section{REFERENCES}

1. King, L. B., Gallimore, A. D., AIAA Paper 96-2712, (1996).

2. Hofer, R. R., Walker, M. L. R., Gallimore, A. D., IEPC Paper 01-20, (2001).

3. Manzella, D. H., Sankovic, J. M., AIAA Paper 95-2927, (1995).

4. Gallimore, A. D., Journal of Spacecraft and Rockets, 38 (3), 441-453 (2001).

5. Semenkin, A., Kim, V., Gorshokov, O., Jankovsky, R., IEPC Paper 2001-070, (2001).

6. Jankovsky, R. S., Jacobson, D. T., Mason, L. S., Rawlin, V. K., Mantenieks, M. A., Manzella, D. H., Hofer, R. R., Peterson, P. Y., AIAA Paper 2001-3888, (2001).

7. Boyd, I. D., Journal of Spacecraft and Rockets, 38 (3), 381-387 (2001).

8. Fife, J. M., Hargus, W. Jr, Jaworske, D. A., Sarmient, C., Mason, L., Jankovsky, R., Snyder, J. S., Malone, S., Haas, J., Gallimore, A., AIAA Paper 2000-3521, (2000).

9. Hofer, R. R., Peterson, P. Y., Gallimore, A. D., IEPC Paper 01-045, (2001).

10. de Grys, K. H., Tilley, D. L., Aadland, R. S., AIAA Paper 99-2283, (1999).

11. Walker, M. L. R., Hofer, R. R., Gallimore, A. D., AIAA Paper 2002-4253, (2002).

12. Walker, M.L.R., Gallimore, A.D., Cai, C.P., and Boyd, I.D., AIAA Paper 2002-3815, (2002).

13. Dushman, S., Scientific Foundations of Vacuum Technique, Vol. 4, Wiley, New York, 1958.

14. Oh, D.Y. and Hastings, D.E., AIAA Paper 96-3196, (1996).

15. VanGilder, D.B., Boyd, I.D. and Keidar, M., Journal of Spacecraft and Rockets, 37, 129-136 (2000).

16. Boyd, I.D., Journal of Propulsion and Power, 16, 2000, 902-909 (2000).

17. Bird, G.A., Molecular Gas Dynamics and the Direct Simulation of Gas Flows, Oxford University Press, 1994.

18. Birdsall, C.K. and Langdon, A.B., Plasma Physics Via Computer Simulation, Adam Hilger, 1991.

19. Dalgarno, A., McDowell, M.R.C. and Williams, A., Proceedings of the Royal Society, 250, 411-423 (1958).

20. Miller, S., Levandier, D.J., Chiu, Y., and Dressler, R. A., Journal of Applied Physics, 99, 984-990 (2002).

21. Ketsdever, A. D., Journal of Spacecraft and Rockets, 38, 400-410 (2001).

22. Gulczinski, F.S., Ph.D. Dissertation, Department of Aerospace Engineering, University of Michigan, Ann Arbor, 1999. 